\title{
Neutron imager with micro channel plates (MCP) in electrostatic mirror configuration: Experimental test with radiation source
}

\author{
V. Variale \\ Istituto Nazionale Fisica Nucleare, INFN Sezione di Bari, Via Orabona 4, Italy \\ B. Skarbo \\ Budcker Institute, Novosibirsk, Russia \\ Published 10 July 2018
}

\begin{abstract}
The design of a new high-transparency device based on a Micro Channel Plate (MCP) detector was recently proposed for monitoring the flux and beam spatial profile of neutron beams. The proposed device consists of a very thin aluminum (Al) foil (with a ${ }^{6} \mathrm{Li}$ deposit) placed in the neutron beam and an MCP detector equipped with a phosphor-screen readout linked to a chargecoupled device (CCD) camera outside the neutron beam. A critical feature of this device is that it uses an electrostatic mirror to minimize the perturbation of the neutron beam (i.e., absorption and scattering). It can be used at existing neutron time-of-flight (n_TOF) facilities (in particular at the $\mathrm{n} \_$TOF facility at CERN) for monitoring the flux and spatial profile of neutron beams in the thermal and epithermal region. The experimental tests conducted for this study using a radioactive source to determine the behavior of the electrostatic mirror behavior will be presented and discussed in this paper.
\end{abstract}

Keywords: Beam monitor; neutron detectors.

\section{Introduction}

In recent years, Micro Channel Plate (MCP) devices have been largely employed both for detection of ionizing radiation and as image intensifiers. It was also found that MCPs can be applied in neutron detection and imaging with many advantages. ${ }^{1-3}$ Recently the design of a new high-transparency device based on an MCP detector was proposed for monitoring the flux and spatial profile of a neutron beam. The device's design details are depicted in Fig. 1, and are discussed below and in Ref. 4.

The device consists of an $\mathrm{Al}$ foil with a ${ }^{6} \mathrm{Li}$ deposit (used as a slow-neutron converter) placed in the beam and an MCP detector equipped with a phosphor-screen readout linked to a CCD camera placed outside the beam. The reaction ${ }^{6} \mathrm{Li}(\mathrm{n}, \alpha) \mathrm{t}$ increases the likelihood that slow neutrons hitting the ${ }^{6} \mathrm{Li}$ atoms will produce alpha $(\alpha)$ and triton $(t)$ ions with $2 \mathrm{MeV}$ and $2.7 \mathrm{MeV}$, respectively. At those energies, the $\alpha$ and $t$ particles have (in the $\mathrm{Al}$ foil) a range of $5 \mu \mathrm{m}$ and $30 \mu \mathrm{m}$, respectively. Then, by using an Al foil with a thickness of $14 \mu \mathrm{m}$, all of the $\alpha$ particles can be stopped, and all of the $t$ particles are transmitted.

This is an Open Access article published by World Scientific Publishing Company. It is distributed under the terms of the Creative Commons Attribution 4.0 (CC-BY) License. Further distribution of this work is permitted, provided the original work is properly cited. 
All of the $t$ particles then exit from the Al foil and emit secondary electrons (SEs), which are in turn accelerated by the nearby grid and reflected (at a 90-degree angle) toward the MCP by the other grids angled at $45^{\circ}$ (electrostatic mirror). The SEs impinging on the MCP detector's inner channels generate an electron avalanche. Behind the MCP (in chevron configuration) there is the signal read-out system, a phosphor screen linked to a CCD camera where the beam image is recorded.

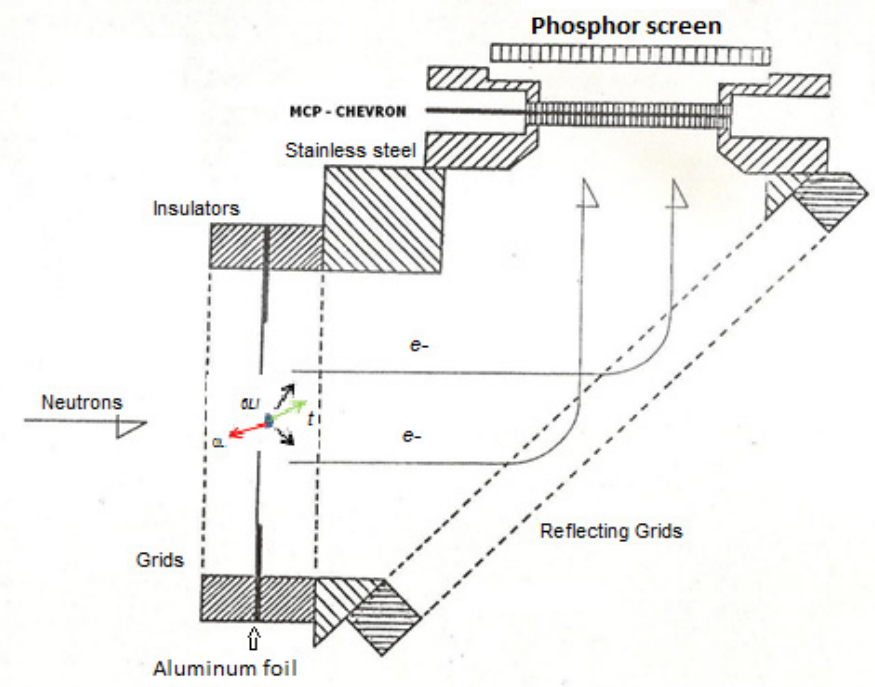

Fig. 1. Schematic diagram of the neutron-beam monitor in the electrostatic-mirror configuration.

The experimental tests conducted for this study using a radioactive source to determine the behavior of the electrostatic mirror will be presented and discussed in this paper.

\section{The Neutron Beam Monitor}

The main feature of the device is based on the MCP's detection of the SEs produced in the slow-neutron converter. The slow neutrons that hit the converter react with ${ }^{6} \mathrm{Li}$ atoms and produce $\alpha$ and $t$ particles. Since the range of the $\alpha$ particles produced is much less than the Al foil thickness, all the $\alpha$ particles will be stopped while all the $t$ particles, which have a longer range, will pass through. The $t$ particles exiting the Al foil produce SEs with an energy distribution peaked around $1-2 \mathrm{eV}$ and an angular distribution that is dependent upon the exit angle of the $t$ particles. The SEs are quickly accelerated by the nearby grid and then deflected at $90^{\circ}$ (as shown in Fig. 1) toward the MCP and the phosphor screen, where they generate light points (LPs) that are "seen" and recorded by a CCD camera. 


\section{Experimental Set-Up}

The experimental set-up used for testing the neutron monitor with the radiation source is shown in Fig. 2. It was installed in a small vacuum chamber. Due to the small size of the chamber's flanges, the electrostatic mirror support bars could not be mounted on the same flange as the MCP assembly. The radioactive source used for the test was ${ }^{241} \mathrm{Am}$, which produces $\alpha$ particles of about $5.5 \mathrm{MeV}$ with an activity of $3 \mathrm{kBq}$. The source particles, whose ranges are longer than the thickness of the Al foil, were able to exit the foil, producing SEs that could be accelerated toward the MCP and the phosphor screen where the MCP output signals produce LPs. The CCD camera used for the monitor read out was a Hamamatsu ORCA-05 with a $55 \mathrm{~mm}$ focal length lens. A 2.9 aperture setting was used. The camera's total pixel number is $1344(\mathrm{H}) \times 1024(\mathrm{~V})$, with an image-field size of $8.67 \mathrm{~mm}$ (horizontal) $\times 6.6 \mathrm{~mm}$ (vertical).

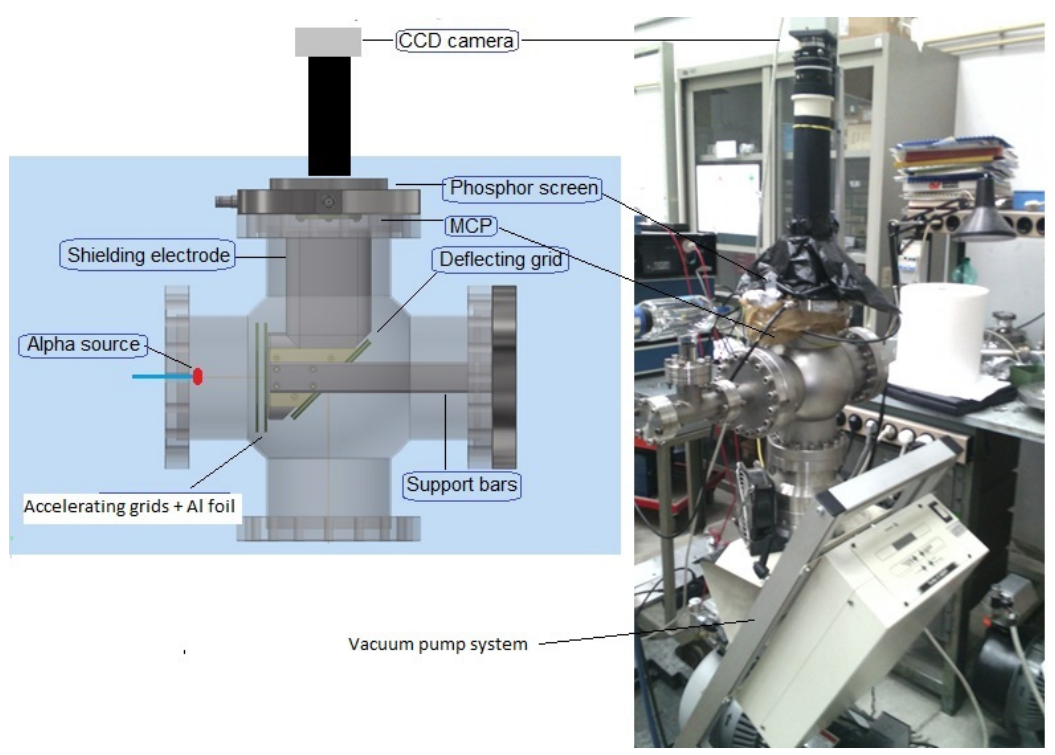

Fig. 2. Experimental set-up used for testing the neutron monitor with the radiation source.

Each CCD pixel has a per-side size of $6.45 \mu \mathrm{m}$. The camera's software (Hamamatsu's HCImageLive) enabled it to achieve an increase in on-screen resolution of each LP of about 5 pixels vertically and horizontally (see Fig. 3). The limit of the LP position resolution can be evaluated by considering the full width at half maximum (FWHM) of the signal, about $14 \mu \mathrm{m}$, which is lower than the phosphor grains' resolution listed in the Hamamatsu MCP assembly manual. For this study, the maximum exposure time of the camera was only one second. In order to obtain measurements of higher statistical quality, 100 snapshots of one second each were recorded in succession for each 
measurement. In our measurements, each pixel reached the saturation for a luminosity of 4095 (a.u.).

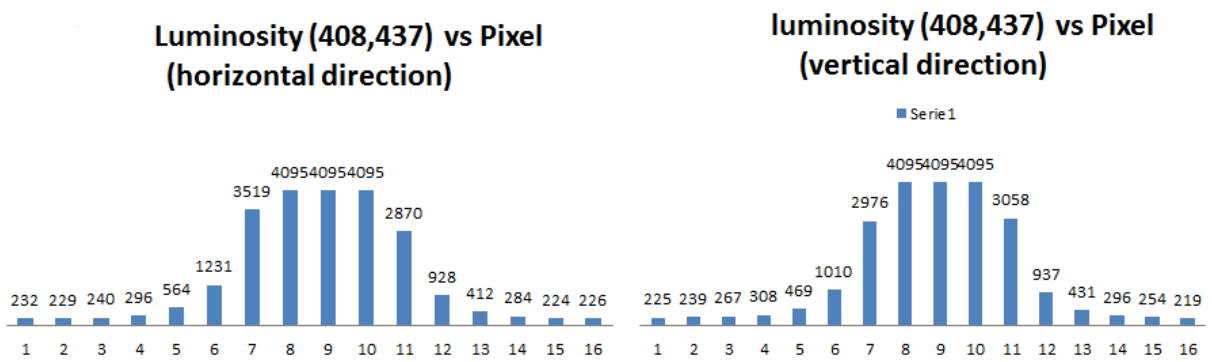

Fig. 3. The luminosity of the LP placed in the position $(408,437)$ is shown with up to 8 pixels around it.

\section{Experimental Test Measurements}

The position of the $\alpha$ source in the vacuum chamber is shown in Fig. 2 above. The test measurements (displayed on camera snapshots of one second each) compared favorably with the MCNPX simulation results cited in Ref. 5. Figure 4 shows the signal and the background image after a time integration of $100 \mathrm{~s}$. In a), the CCD camera signal for the case of electrostatic mirror switched off (that is $\mathrm{V}_{\mathrm{f}}=\mathrm{V}_{\mathrm{g}}=0 \mathrm{~V}$ ) is shown; in $\mathrm{b}$ ), the signal for the case of electrostatic mirror switched on $\left(\mathrm{V}_{\mathrm{f}}=\mathrm{V}_{\mathrm{g}}=-1 \mathrm{kV}\right)$ is shown.

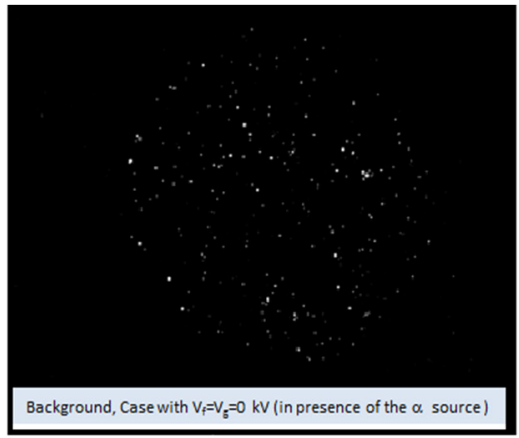

a)

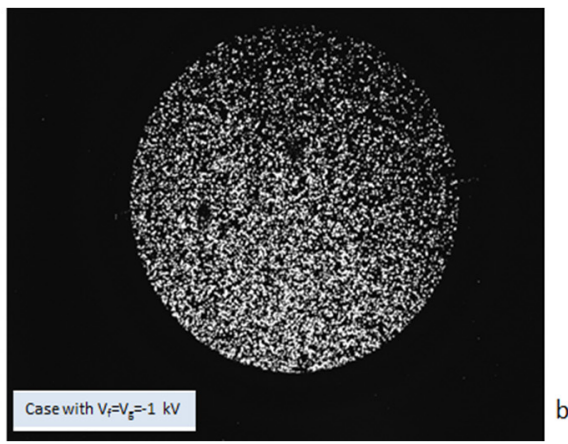

Fig. 4. Camera snapshots of $1 \mathrm{~s}$ that show measurements for the cases: a) background and b) $V_{\mathrm{f}}=\mathrm{V}_{\mathrm{g}}=-1 \mathrm{kV}$.

Measurements of $\mathrm{V}_{\mathrm{f}}=\mathrm{V}_{\mathrm{g}}=-2 \mathrm{kV}$ were obtained because higher accelerating voltages for the SEs could improve the beam image, as cited in Ref. 4. Furthermore, the MCP detection efficiency for electrons reaches a maximum in the energy range of $1-2 \mathrm{keV}$. A further increase of $\mathrm{V}_{\mathrm{g}}$ induced a corona effect around the deflecting grid wires, as shown in Ref. 5. The deflecting voltage changes, however, should also anticipate or postpone the deflection of the SE trajectories toward the MCP and phosphor screen (see Fig. 1), 
resulting in an image shift. Measurements with different $\mathrm{V}_{\mathrm{g}}$ values were obtained; the $\alpha$ source images are shown in Fig. 5. For $\mathrm{V}_{\mathrm{g}}=-800 \mathrm{~V}$, the LPs do not have a very flat distribution. In fact, apart from the two visible holes due to defects in the phosphor coating, a kind of shadow is visible in the upper-right quadrant of the source image. The shadow practically disappears in the case where $\mathrm{V}_{\mathrm{g}}=-1.8 \mathrm{kV}$. This study also used $\mathrm{V}_{\mathrm{g}}$ values higher than $2.0 \mathrm{kV}$, although those values generated electrons by corona effect, which artificially increased the image luminosity, as cited in Ref. 5. The far-left image of Fig. 5 depicts the case with $\mathrm{Vg}=-2.5 \mathrm{kV}$. A side-effect of the higher luminosity due to the corona effect, which appears as a slight shadow (lower LP density), is visible in the left part (instead of the right part) of the source image.
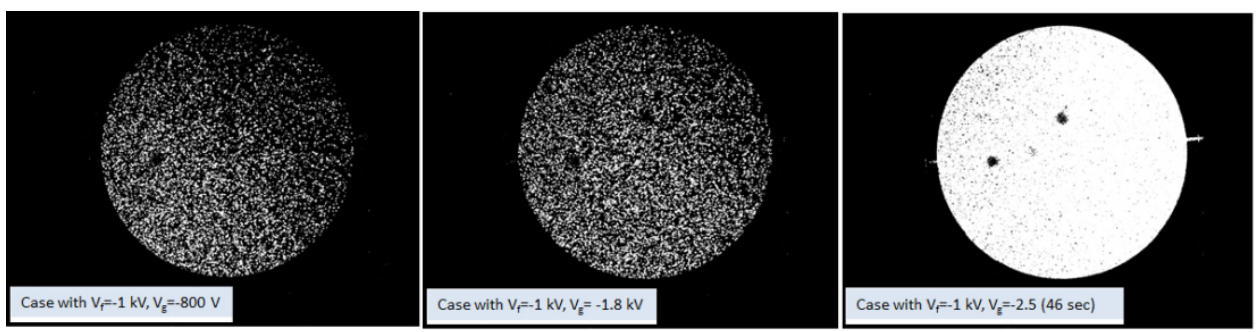

Fig. 5. The $\alpha$ source images for the different $V_{g}$ values.
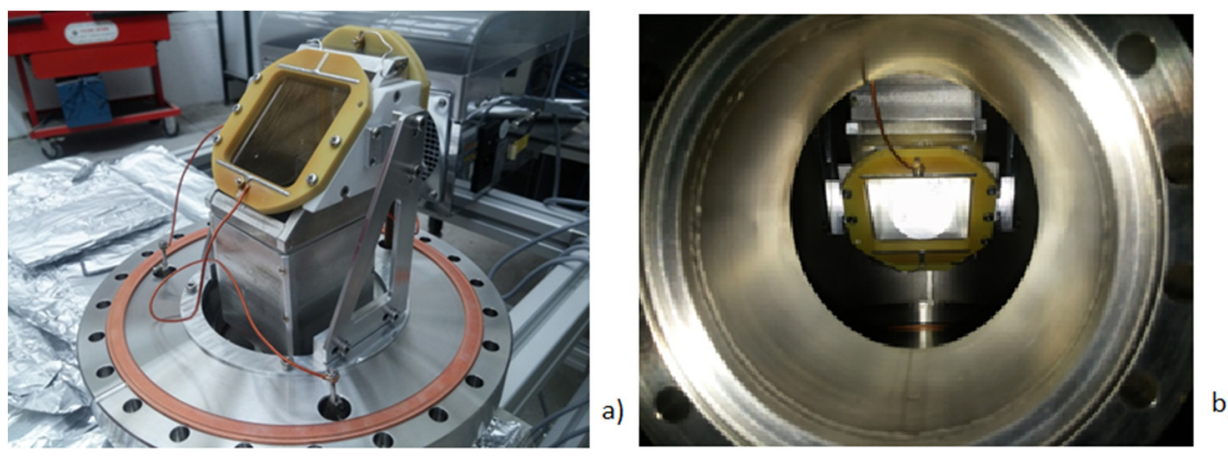

Fig. 6. a) New electrostatic mirror support mounted on the larger flange; b) the electrostatic mirror mounted in the vacuum chamber.

The monitor test on the neutron beam is planned to take place before the end of the year. Since the main feature of the monitor is its high transparency (very low beam perturbation), the experimental set-up used for the test with the source was not suitable. The MCP device was installed in a small vacuum chamber with very small flanges, so the electrostatic mirror support bars could not be mounted on the same flange as that of the MCP assembly. A new vacuum chamber with larger flanges was acquired and it provided enough room to mount the electrostatic mirror of the monitor on the same MCP flange, as shown in Fig. 6. The monitor mounted in the new vacuum chamber is also shown in 
Fig. 6b. Note that on the beam axis, there is only the converter foil and the deflecting grid without other encumbrances.

A test of the beam monitor with the new electrostatic mirror support on the MCP assembly flange (Fig. 6a) was conducted; the measurement results are shown in Fig. 7. There is a noticeable difference between the two $\alpha$ images of Fig. 7, although the grid voltage used was the same. That difference is due to the different positions of the $\alpha$ source with respect to the Al foil used in the two measurements. In fact in the new vacuum chamber, the distance to the $\alpha$ source was about $2 \mathrm{~cm}$, while in the old chamber it was about $7 \mathrm{~cm}$.
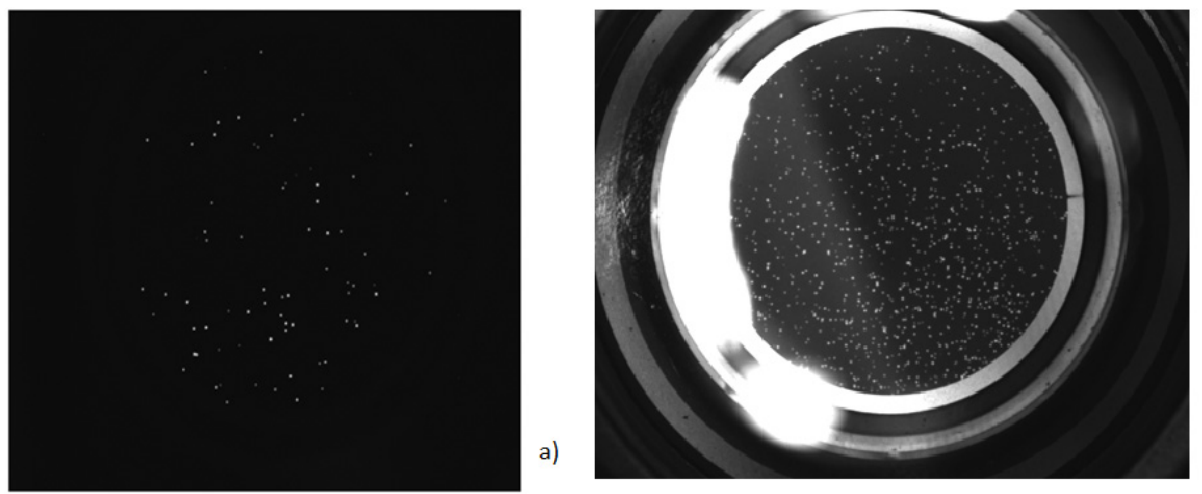

b)

Fig. 7. CCD camera snapshot, $1 \mathrm{~s}$ of exposure time: a) $\alpha$ source image shot in the previous vacuum chamber where $\left.V_{f}=V_{g}=-1 \mathrm{kV} ; b\right) \alpha$ source image taken in the new vacuum chamber $\left(V_{f}=V_{g}=-1 k V\right)$.

\section{Conclusion}

The high-transparency neutron monitor tests were conducted with an $\alpha$ source by using two different vacuum chambers. A larger vacuum chamber was required for mounting the monitor on the same flange as that of the MCP assembly used for the SE detection. To obtain measurements of greater statistical value, the CCD images taken with a onesecond exposure time have been integrated up to $100 \mathrm{~s}$ by using the CCD camera acquisition software (HCImageLive). Testing of the monitor on a neutron beam is planned to take place before the end of this year.

\section{References}

1. G. W. Fraser and J. F. Pearson, Nucl. Instr. and Meth. A293, 569 (1990).

2. O. H. W. Siegmund, A. S. Tremsin, J. V. Vallerga, J. Hull, IEEE Trans. Nucl. Sci. NS-48, 430 (2001).

3. A. S. Tremsin, W. B. Feller, R. G. Downing, Nucl. Instr. and Meth. A539, 278 (2005).

4. V. Variale, Physics Procedia 66, 242 (2015).

5. V. Variale, B. Skarbo, Physics Procedia, in pub. (2017). 